\title{
Extensor Carpi Radialis Brevis: Review of Anatomy and Clinical Significance to Orthopedics
}

Jennifer L Smith a*, Jacob B Stirton a, Nabil A Ebraheim a

The University of Toledo College of Medicine and Life Sciences, Department of Orthopaedic Surgery, 3000 Arlington Avenue, Toledo, OH 43614, USA.

* Corresponding Author: Jennifer L Smith a, The University of Toledo College of Medicine and Life Sciences, Department of Orthopaedic Surgery, 3000 Arlington Avenue, Toledo, OH 43614, USA. E-mail:Jennifer.Smith9@ rockets.utoledo.edu

Received date: September 19, 2019; Accepted date: September 30, 2019; Published date: October 11, 2019

Citation: Jennifer LS., Jacob BS., Nabil AE. (2019) Extensor Carpi Radialis Brevis: Review of Anatomy and Clinical Significance to Orthopedics . J. Orthopaedics and Surgical Sports Medicine, 2(1); DOI: 10.31579/2641-0427/017

Copyright: (C) 2019, Jennifer L Smith. This is an open-access article distributed under the terms of the Creative Commons Attribution License, which permits unrestricted use, distribution, and reproduction in any medium, provided the original author and source are credited.

\begin{abstract}
The extensor carpi radialis brevis (ECRB) muscle is an integral extensor and abductor of the wrist. It originates from the lateral epicondyle of the humerus, laying deep to the extensor carpi radialis longus and extensor digitorum communis, and superficial to the supinator. Insertion occurs at the base of the third metacarpal. The radial nerve or a derivative supplies innervation. Its significance in orthopedics is highlighted by its involvement in multiple surgical approaches, such as the Thompson and Kaplan approaches for exposure of the radius, as well as its association with several routinely observed pathologies. Many of the associated syndromes, such as lateral epicondylitis, arise from repetitive gripping motions or overuse and are frequently seen in the orthopedic clinic. This review seeks to provide a comprehensive summary of the relevance of the ECRB to the orthopedic setting to broaden knowledge of its anatomy and increase recognition and proper management of associated pathologies.
\end{abstract}

Key Words: extensor carpi radialis brevis; radial nerve; thompson approach; lateral epicondylitis; overuse injuries

\section{Introduction}

The extensor carpi radialis brevis (ECRB) is a muscle spanning the dorsal aspect of the forearm primarily responsible for extension and abduction of the wrist. It is relevant to orthopedic surgeons for a variety of reasons, including its association with a number of common pathologies and surgical approaches. The aim of this review is to summarize pertinent topics and to provide surgeons with an inclusive and up-to-date resource on the clinical significance of the ECRB. Subjects discussed include anatomy of the ECRB; the Thompson, Kaplan, Cadenat and Gschwend approaches for exposure of the radius; radial tunnel syndrome (posterior interosseous nerve entrapment); lateral epicondylitis (tennis elbow); radial nerve palsy; and intersection syndrome. These foci were selected based on a critical survey of the literature in PubMed. To the authors' knowledge, no similar review exists at the presen time.

\section{Main Text}

\subsection{Anatomy of the ECRB}

Alongside the ECRL, the ECRB is the main extensor of the wrist, originating at the lateral epicondyle of the humerus and following the dorsal surface of the forearm to its insertion in the base of the third metacarpal, as seen in Figure 1. 


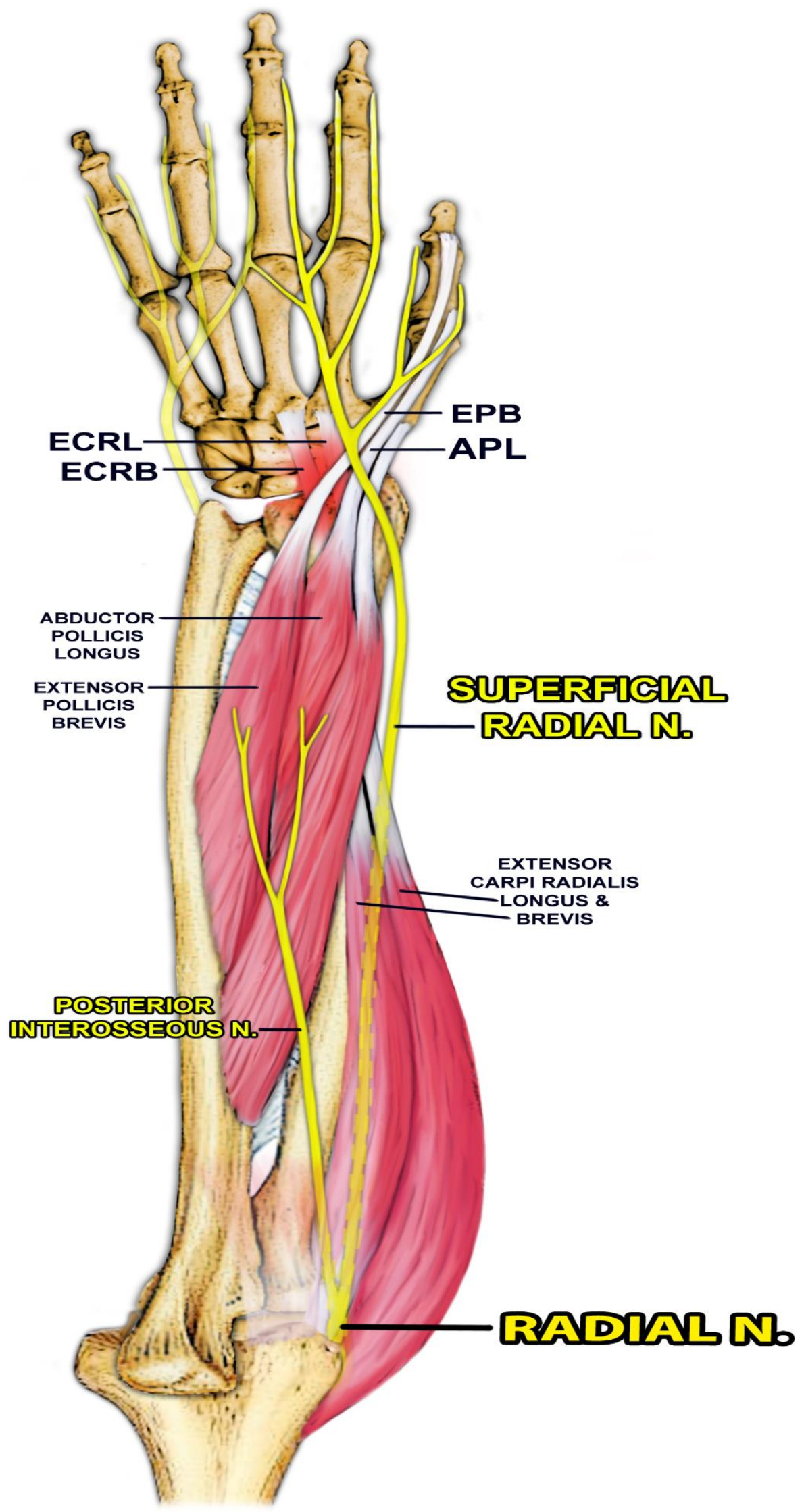

Figure 1: Anatomy of the ECRB, The ECRB originates at the lateral epicondyle of the humerus and inserts on the base of the third metacarpal, lending to its function as a major extensor and abductor of the hand at the wrist. 
Accounts of origins other than the lateral epicondyle have been reported, including the deep capsuloligamentous structures (i.e. radial collateral ligament or lateral ulnar collateral ligament of the elbow joint) and adjacent intermuscular septum [1,2]. Differentiation is often difficult, even with distal to proximal dissection, as the ECRB tendon merges with the tendon of the extensor digitorum communis (EDC) proximally $[2,3]$. Tendons of the extensor carpi ulnaris (ECU) and extensor digiti minimi (EDM) also attach in the surrounding area [4].

The ECRB muscle lies deep to the extensor carpi radialis longus (ECRL) and EDC [2] and superficial to the supinator. It has been shown to be innervated by either the deep posterior interosseous nerve (PIN) or superficial sensory branch of the radial nerve, which are the bifurcations of the radial nerve proper at the level of the lateral epicondyle [2,5]. A direct branch of the radial nerve proper has also been reported as supplying the ECRB [5]. The ECRL is also innervated by the main radial nerve. It assists the ECRB in wrist extension via its origin from the lower third of the lateral supracondylar ridge of the humerus and lateral intermuscular septum, and through its insertion in the posterior base of the second metacarpal.

\subsection{Surgical Approaches For Exposure Of The Radius Involving The Ecrb}

Various surgical approaches exist for exposure of the radius involving the ECRB [6]. The posterior or dorsal approach, also known as the Thompson approach, is often used for fracture fixation of the proximal radius, treatment of nonunion or delayed union, tumor biopsy and treatment, nerve exploration, as well as for treatment of radial tunnel release [7, 8]. As with other safe operative approaches, it uses internervous planes, meaning dissection occurs between muscles innervated by different nerves [5]. Proximally, as depicted in Figure 2, the Thompson approach employs the plane between the ECRB and EDC, and distally, between the ECRB and extensor pollicis longus (EPL) [5, 6].

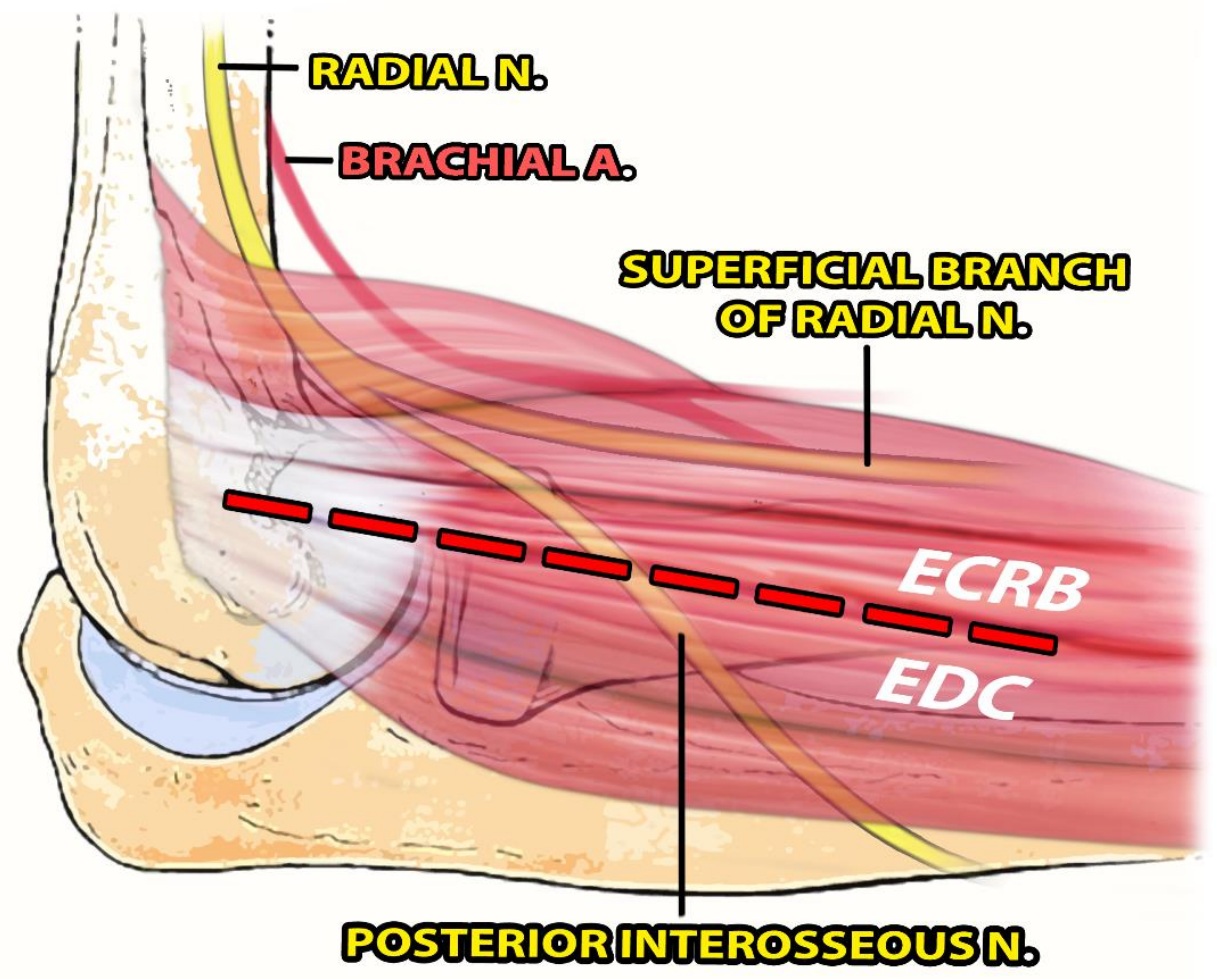

Figure 2: Thompson Approach, Proximally, the Thompson approach employs the internervous plane between the ECRB and EDC. This procedure allows for broad exposure of the radius, while greatly reducing the risk of muscle denervation.

This methodology allows for visualization and protection of the PIN [8], while permitting extensive mobilization of muscles, resulting in a large area of exposure with greatly reduced possibility of muscle denervation [5].

The Kaplan approach to the lateral elbow is another method involving the ECRB, which is utilized for exposure of the radial head [9]. Indications include proximal radial head fractures, capitellum fractures, PIN decompression, proximal radial tumors, and irrigation and drainage of a septic elbow. It also employs dissection of the interval between the ECRB and the EDC, although it lacks popularity among surgeons due to its proximity to the PIN [9].

Two less well-described surgical approaches to the elbow and proximal forearm involving the ECRB include the Cadenat and Gschwend approaches. The Cadenat approach is classified as a lateral strategy and uses the tissue plane between the ECRB and the ECRL [10]. The Gschwend approach is also a lateral method. It involves the tissue planes between the ECU and aconeus, and between the EDC, ECRL and ECRB [11].

\subsection{Pathologies associated with the ECRB}

\subsubsection{Radial Tunnel Syndrome}

Found spanning from the radiocapitellar joint to the distal edge of the supinator muscle, the radial tunnel is a space in the dorsal forearm that is bordered laterally by the ECRB, among other muscles [7]. Although entrapment of the radial nerve in this tunnel may occur for a variety of reasons, impingement from the medial border of the ECRB is an important etiology to consider [7]. Observed in $40.2 \%$ of cadavers in a particular study [1], the ECRB may have a tendinous arch on its medial edge, as well 
as an aponeurosis at its undersurface, possibly responsible for compression of the nerve [12]. Entrapment may be brought about by repetitive activity of the index and middle fingers, such as with frequent computer use [7]. Symptoms consist of pain at the proximal forearm or lateral elbow, and occasionally the back of the hand, leading to a diagnosis of radial tunnel syndrome. This disorder is also referred to as posterior interosseous nerve (PIN) entrapment and radial pronator syndrome.

Significant to the discussion of this pathology, the presentation of radial tunnel syndrome is frequently confused with lateral epicondylitis (tennis elbow) [7], also associated with the ECRB and discussed below. Additionally, they may occur simultaneously in approximately $5 \%$ of cases, further complicating diagnoses, with symptoms of lateral epicondylitis being aggravated by those of radial tunnel syndrome [7]. They can be distinguished based on the location of the point of maximal tenderness where radial tunnel syndrome is typically more distal than lateral epicondylitis. Radial tunnel syndrome is most tender 3-5 cm distal to the lateral epicondyle where lateral epicondylitis is most tender to palpation a few millimeters distal to the lateral epicondyle at the origin of the ECRB.

Management of radial tunnel syndrome is most often via conservative methods. These include splinting, NSAIDs, modification of activity, and stretching [7]. If after three months symptoms do not improve, surgical intervention is indicated. Surgery is a last resort as surgical decompression has had inconsistent outcomes [7]. Applicable surgical options for decompression include the Thompson approach, which involves a plane following the ECRB as explained above; the dorsal approach between the brachioradialis and wrist extensors; the anterior approach, which allows for exposure of the radial nerve both proximal and distal to the elbow; and the transmuscular brachioradialis-splitting approach [13].

\subsubsection{Lateral Epicondylitis (Tennis Elbow)}

Lateral epicondylitis, also known as tennis elbow, is a pathology associated with the ECRB that is more common than radial tunnel syndrome. It occurs at a rate of $1-3 \%$ annually versus $0.003 \%$

$[1,7,14]$ for radial tunnel syndrome. Both males and females are affected equally, with most patients between the ages of 35 and 55 years $[1,4]$. Development may be attributed to prolonged or repetitive gripping motions, with reports of incidence as high as $15 \%$ among workers in industries that require corresponding manual labor tasks [14]. Symptoms include pain in the area of the lateral epicondyle and proximal forearm, which is heightened by contraction of the forearm extensors [15-17].

Although other tendons may be involved, the ECRB is the structure most associated with lateral epicondylitis. Similar to radial tunnel syndrome, it may be caused by increased pressure on the PIN via the tendinous arch of the ECRB [1]. Compression of the radial nerve proper or other branches may also be neurogenic triggers [1]. Alternatively, repetitive strain has been cited as instigating microdamage to the ECRB tendon [14, 15], with hallmarks of tendinosis apparent, such as disorganization of collagen fibers, vascular hyperplasia, and shifting cell populations [4]. Important to note, although tennis elbow is labeled as a tendinitis, signaling a case of inflammation, the disorder is not primarily an inflammatory reaction [15].

One of the most significant risk factors for lateral epicondylitis is rotator cuff pathology, as they are both enthesopathies [18], in that there are shared microvascular and cellular pathological processes [18-21]. De Quervain's syndrome, carpal tunnel syndrome, and trigger finger are also significantly associated with tennis elbow [18]. Other risk factors noted in the literature include: obesity [18, 22, 23], current or previous tobacco use, and untreated diabetes mellitus [12]. Rheumatoid arthritis was not found to be associated nor was alcohol consumption [18].

Treatment of lateral epicondylitis is most often successful via traditional conservative means, such as rest, NSAIDs, counterforce bracing, physical therapy (usually first-line of treatment), and corticosteroid injections, as the condition is commonly self-limiting $[14,15]$. It is important to note, however, that the long-term effectiveness of corticosteroids has been questioned. Although corticosteroids are thought to decrease levels of Substance $P$ in the body, providing a mechanism of pain relief [15], literature suggests this short-term alleviation may be outweighed by deleterious consequences. Despite their use for more than a century, corticosteroids have been associated with increased risk of requiring surgery $[14,24]$, decreased tenocyte replication, and collagen production [15]. If more conservative approaches are desired, ultrasound waves and radiofrequency probes are available techniques [15].

If conservative management fails, lateral epicondylitis can also be treated with intermediately invasive options, several of which have demonstrated beneficial outcomes. These include autologous blood preparation injections [15] and platelet-rich plasma injections [4], and use of cell populations for tendon repair, such as multipotent stem cells, skin fibroblasts, and tenocytes [15]. Options still under investigation include Botulinum toxin A, prolotherapy, and delivery of $\mathrm{NO}$ [15].

In approximately $4-11 \%$ of cases, more conservative treatments do not yield improvement after 6 months, and surgical intervention is recommended $[2,14,15]$. Three methods exist, in which the origin of the ECRB is released and debrided: open, arthroscopic, and percutaneous [2, 25]. Each has been described with good outcomes and minimal complications $[2,14,25]$, although open release may warrant a higher risk of infection [25]. Complications that may be encountered include nerve injury, particularly to the radial nerve (radial nerve palsy), and elbow instability [2]. Due to their proximity, as observed in Figure 3, overly aggressive release of the ECRB tendon can disrupt the lateral ulnar collateral ligament resulting in posterolateral rotatory instability elbow (PRLI) [2]. 


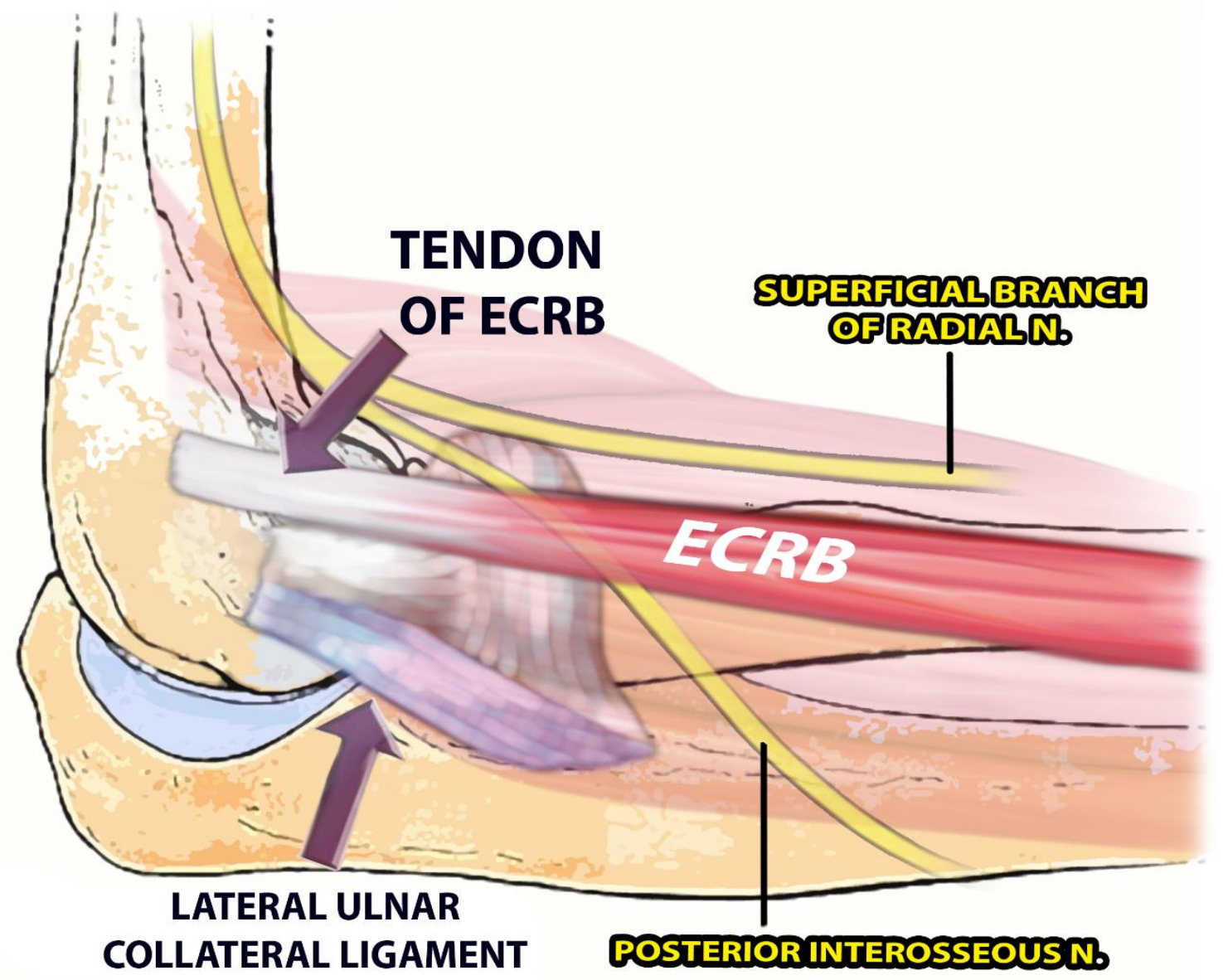

Figure 3: Relationship of the Ulnar Collateral Ligament to the ECRB Tendon, The ulnar collateral ligament lies immediately deep to the ECRB. Risk of posterolateral rotary instability may therefore occur with overly aggressive release of the ECRB tendon during surgical management of lateral epicondylitis.

In a study by Degen et al., risk factors for revision surgery included $<65$ years of age, male gender, morbid obesity, tobacco use, and inflammatory arthritis [14]. Three or more steroid injections preoperatively was the most significant risk factor [14].

\subsubsection{Intersection Syndrome}

Another condition involving the ECRB is intersection syndrome, which has been found to be more common in men and in the dominant hand [26]. The incidence of this syndrome is believed to be underreported, with most cases going unrecognized, resolving quickly on their own, or responding rapidly to conservative treatment [26-28]. Repetitive dorsiflexion and radial deviation of the wrist appears to be the most common cause, associated with activities such as manual farming and construction labor, various racquet sports, rowing, and weightlifting, for example [26,27,29].

The syndrome is a non-infectious inflammatory process involving the muscle bellies or tendons of the abductor pollicis longus (APL) and extensor pollicis brevis (EPB) of the first dorsal compartment of the wrist and the tendons of the ECRB and ECRL of the second dorsal compartment of the wrist [28-30], Figure 4. 


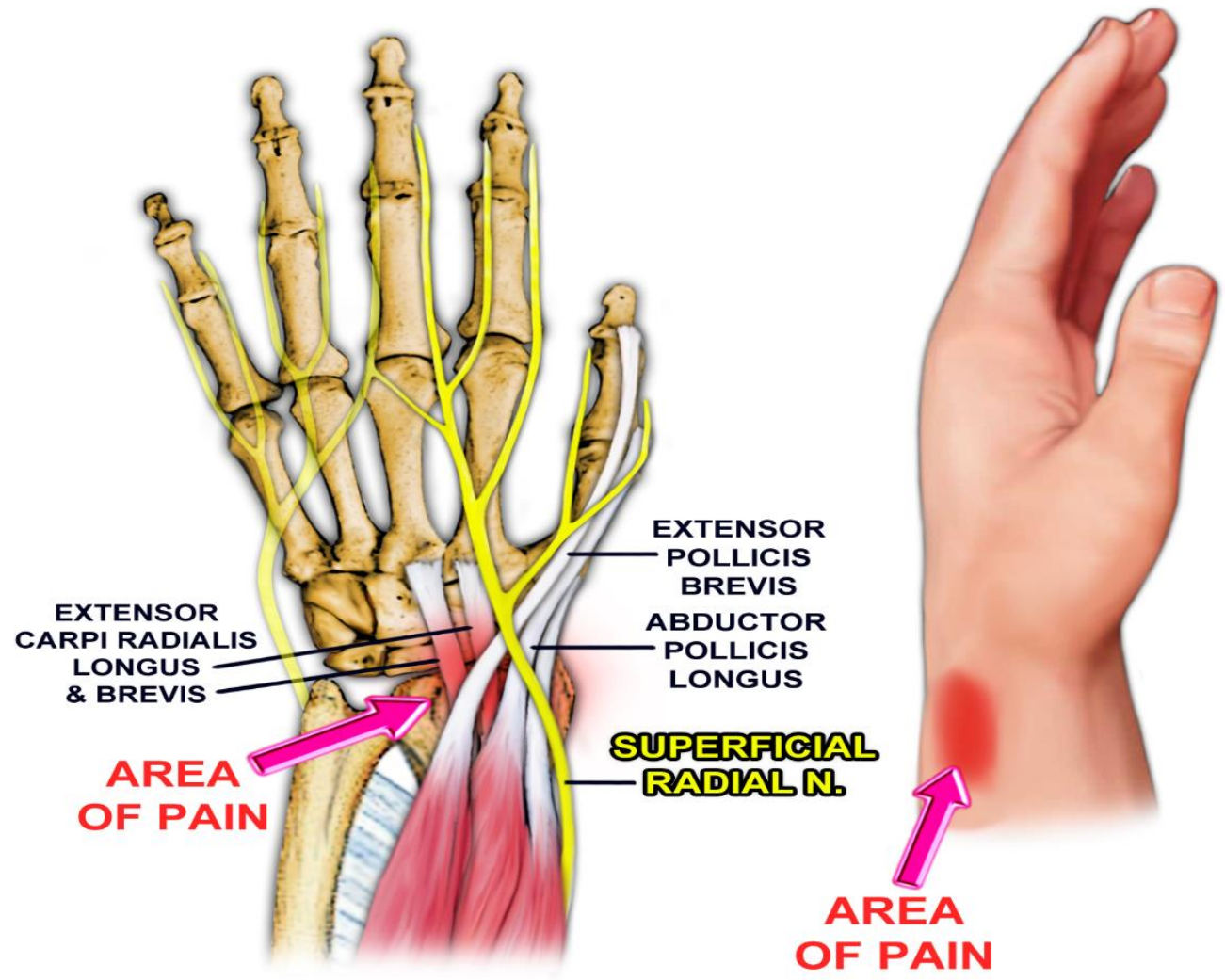

Figure 4: Intersection Syndrome, Intersection syndrome involves the muscle bellies or tendons of the APL and EPB of the first dorsal compartment of the wrist and the tendons of the ECRB and ECRL of the second dorsal compartment of the wrist. It is an inflammatory condition attributed to friction between the structures of the two compartments and tightness of the sheaths surrounding the ECRB and ECRL.

Symptoms, including pain, swelling, erythema, edema, tenderness to palpation, and crepitance with flexion and extension of the wrist, are found at the site where the the APL and EPB cross over the ECRB and ECRL on the dorsal aspect of the wrist near the radial styloid [28-30] (FGIRUE). This pathology is hypothesized to arise for two main reasons: friction between the components of the two compartments due to hypertrophy of the bellies of the APL and EPB, and entrapment from stenosis as a result of swelling from tightness of the sheath of the ECRB and ECRL tendons $[28,30]$.

Differential diagnoses include tenosynovitis (particularly de Quervain's) of the first, second, and third dorsal compartments, blunt local trauma, Wartenberg's syndrome (entrapment of dorsal radial sensory nerve), ganglion cyst, and non-specific cellulitis [27, 28, 30]. However, intersection syndrome is the only binocular tenosynovitis of the hand, thus the presence of fluid within the two extensor compartments as visualized with MRI or ultrasound should yield the correct diagnosis [27, $28,31]$.

Treatment is frequently successful with conservative measures, including rest, activity modification, and splinting [27, 29]. As of June of 2017 , there was no evidenced-based rehab protocol applicable for intersection syndrome [29]. If symptoms continue for more than 2 to 3 weeks, corticosteroid injection is a recommended next step [27, 29]. If all nonoperative options fail to provide relief, surgery with tenosynovectomy and fasciotomy may be done to decompress the area of intersection [27].

\subsubsection{Radial Nerve Palsy}

Radial nerve palsy results in losses such as extension of the wrist (via the ECRB and ECRL), finger extension, and thumb extension/radial abduction, with the greatest functional deficit being the inability to grip [32]. Colloquially, the resulting syndrome is called "wrist drop". Though the radial nerve recovers more successfully after repair than any other nerve in the upper extremity, it may require greater than 6 months [32-35]. To compensate for this prolonged loss of function, the common treatment of radial nerve palsy is tendon transfer [36]. Tendon transfer allows for preservation of function of the wrist and hand; it acts as a substitute for the paralyzed muscle during nerve regeneration and stimulates re-education of sensibility [32, 35]. Further, it avoids external splinting, which patients have described as hindering their daily routine, decreasing adherence [32, 37]. Tendon transfer is also applicable in situations where the outcome of nerve repair is poor or in which a lesion is irreparable [32].

Most commonly, the insertion of the pronator teres (PT) is relocated to the ECRB to reestablish extension at the wrist [32, 33, 38, 39], acting as an internal splint. The released PT muscle is passed deep to the brachioradialis and is interwoven with sutures to the tendon of the ECRB [32]. Studies conducted on the PT have shown that it is quite analogous in architecture and mechanical properties to the ECRB, with a force potential similar to the ECRB and a low difference index $[38,40]$. While this tendon transfer is the conventional method used and is successful at restoring extension of 
the wrist, a resulting loss of muscle strength and the ability to pronate have been detailed [32].

Use of autologous nerve grafts is another practical means for managing radial nerve palsy, specifically median nerve transfer to the radial nerve [36, 41, 42]. As one example, Ray and Mackinnon (2011) investigated the use of redundant median nerve branches as donor nerves, with a median nerve branch to the flexor digitorum superficialis (FDS) transferred to the ECRB nerve and the flexor carpi radialis nerve to the PIN to restore wrist extension [36]. This technique is considered when large, segmental gaps are present in the nerve preventing reconstruction or in the case of documented complete palsy in which there is no possibility of recovery [43].

\section{Conclusion}

Knowledge of the ECRB is important clinically, as it is involved in many common pathologies of the upper extremity, particularly those resulting from overuse, and the widely-applicable Thompson approach. This article acts as a quick-reference tool for surgeons by consolidating such topics into a brief and comprehensive review.

\section{Acknowledgements}

Not applicable.

\section{Declaration of Conflicting Interests}

The Authors declare that there is no conflict of interest.

\section{Author contributions:}

Conception of the work was led by NAE. JLS collected data, drafted the manuscript, and managed figure creation. JBS critically revised the manuscript. All authors had access to the data and approved the final version for publication.

\section{Funding sources: N/A}

Conflict of interest statement: All authors have no conflicts of interest, financial or otherwise

\section{References}

1. Nayak SR, Ramanathan L, Krishnamurthy A, Prabhu LV, Madhlastha S, et al (2010) Extensor carpi radialis brevis origin, nerve supply and its role in lateral epicondylitis. Surg Radiol Anat. 32:207-211.

2. Pomerantz ML. (2016) Complications of lateral epicondylar release. Orthop Clin North Am. 47:445-469.

3. Greenbaum B, Itamura J, Vangsness CT, Tibone J, Atkinson R (1999) Extensor carpi radialis brevis. An anatomical analysis of its origin. J Bone Surg Br. 81:926-929.

4. Brkljac M, Kumar S, Kalloo D, Hirehal K (2015) The effect of platelet-rich plasma injection on lateral epicondylitis following failed conservative management. J Orthop. 12 Suppl 2:166-170.

5. Klausmeyer MA, Mudgal C (2014) Exposure of the forearm and distal radius. Hand Clin. 30:427-433.

6. Jockel CR, Ziolow DA, Butler RB, Becker EH (2013) Extensile surgical exposures of the radius: a comparative anatomic study. J Hand Surg Am. 38:745-752.

7. Kaswan S, Deigni O, Tadisina KK, Totten M, Kraemer BA (2014) Radial tunnel syndrome complicated by lateral epicondylitis in a middle-aged female. Eplasty. 14:ic44.
8. Cross JD, White JA, Johnson AE, Blair JA, Hsu JR (2011) Comparison of dorsal and volar approaches to the proximal radius. Orthopedics. 34:93.

9. Han F, Lim CT, Lim JC, Tan BH, Shen L et al (2016) Deep branch of the radial nerve in lateral surgical approaches to the radial head - A cadaveric study. Orthop Traumatol Surg Res. 102(4):453-458.

10. Azar FM, Canale ST, Beaty JH (2017) Surgical techniques and approaches. In: Azar FM, Canale ST, Beaty JH, eds. Campbell's operative orthopaedics e-book. 13th ed. Philadelphia, PA: Elsevier

11. Pattern SD, Bain GI, Mehta JA (2000) Surgical approaches to the elbow. Clin Orthop Relat Res. 370:19-33.

12. Vergara-Amador E, Ramirez A (2015) Anatomic study of the extensor carpi radialis brevis in its relation with the motor branch of the radial nerve. Orthop Traumatol Surg Res. 101:909-912.

13. Moradi A, Ebrahimzadeh MH (2015) Jupiter JB. Radial tunnel syndrome, diagnostic and treatment dilemma. Arch Bone Jt Surg. 3(3):156-162.

14. Degen RM, Cancienne JM, Camp CL, Altchek DW, Dines JS et al (2017) Three or more preoperative injections is the most significant risk factor for revision surgery after operative treatment of lateral epicondylitis: an analysis of 3863 patients. J Shoulder Elbow Surg. 26:704-709.

15. Kahlenberg CA, Knesek M, Terry MA (2015) New developments in the use of biologics and other modalities in the management of lateral epicondylitis. Biomed Res Int.

16. Calfee RP, Patel A, DaSilva MF, Akelman E (2008) Management of lateral epicondylitis: current concepts. J Am Acad Orthop Surg. 16:19-29.

17. Ahmad Z, Siddiqui N, Malik SS, Abdus-Samee $M$, Tytherleigh-Strong $\mathrm{G}$ et al (2013) Lateral epicondylitis: a review of pathology and management. Bone Joint J. 95:11581164.

18. Titchener AG, Fakis A, Tambe AA, Smith C, Hubbard RB et al (2013) Risk factors in lateral epicondylitis (tennis elbow): a case-control study. J Hand Surg Eur. 38:159-164.

19. Laban MM, Iyer R, Tamler MS (2005) Occult periarthrosis of the shoulder: a possible progenitor of tennis elbow. Am J Phys Med Rehabil. 84:895-898.

20. Chard MD, Cawston TE, Riley GP, Gresham GA, Hazleman BL (1994) Rotator cuff degeneration and lateral epicondylitis: a comparative histological study. Ann Rheum Dis. 53: 30-34.

21. Kraushaar BS, Nirschl RP (1999) Current concepts review tendinosis of the elbow (tennis elbow). Clinical features and findings of histological, immunohistochemical, and electron microscopy studies. J Bone Joint Surg Am. 8:259-278.

22. Roquelaure Y, Ha C, Rouillon C et al (2009) Risk factors for upper- extremity musculoskeletal disorders in the working population. Arthritis Rheum. 61:1425-1434.

23. Shiri R, Viikari-Juntura E, Varonen H, Heliövaara M (2006) Prevalence and determinants of lateral and medial epicondylitis: A population study. Am J Epidemiol. 164:10651074.

24. Kachooei AR, Talaei-Khoei M, Faghfouri A, Ring D (2016) Factors associated with operative treatment of enthesopathy of the extensor carpi radialis brevis origin. J Shoulder Elbow Surg 25:666-670.

25. Pierce TP, Issa K, Gilbert BT, Hanly B, Festa A, et al (2017) A systematic review of tennis elbow surgery: open versus arthroscopic versus percutaneous release of the common extensor origin. 33:1260-1266.

26. Pantukosit S, Petchkrua W, Stiens SA (2001) Intersection syndrome in Buriram Hospital: a 4-yr prospective study. Am J Phys Med Rehabil. 80:656-661. 
27. Shiraj S, Winalski CS, Delzell P, Sundaram M (2013) Radiologic case study, intersection syndrome of the wrist. Orthopedics. 36:165, 225-227.

28. Giovagnorio F, Miozzi F (2012) Ultrasound findings in intersection syndrome. J Med Ultrason. 39:217-220.

29. Michols N, Bhimji S (2017) Intersection Syndrome. [Updated 2017 May 16]. In: StatPearls [Internet]. Treasure Island (FL): StatPearls Publishing.

30. Descatha A, Laproust H, Roure P, Ronan C, Roquelaure $\mathrm{Y}$ (2008) Is the intersection syndrome an occupational disease? Joint Bone Spine. 75:329-331.

31. Sato J, Ishii Y, Noguchi H (2016) Clinical and ultrasound features in patients with intersection syndrome or de Quervain's disease. J Hand Surg Eur. 41:220-225.

32. Dabas V, Suri T, Surapuraju PK, Sural S, Dhal A (2011) Functional restoration after early tendon transfer in high radial nerve paralysis. J Hand Surg Eur. 36:135-140.

33. Al-Qattan MM (2012) Tendon transfer for radial nerve palsy: a single tendon to restore finger extension as well as thumb extension/radial abduction. J Hand Surg Eur. 37:855-862.

34. Adams J, Wood VE (1981) Tendon transfers for irreparable nerve damage in the hand. Orthop Clin North Am. 12:403432 .
35. Burkhalter WE (1974) Early tendon transfer in upper extremity peripheral nerve injury. Clin Orthop Relat Res. 104:68-79.

36. Ray WZ, Mackinnon SE (2011) Clinical outcomes following median to radial nerve transfers. J Hand Surg Am. 36:201-208.

37. Paternostro-Sluga T, Keilani M, Posch M, Fialka-Moser V (2003) Factors that influence the duration of splint wear in peripheral nerve lesions. Am J Phys Med Rehabil. 82:86-95.

38. Skie MC, Parent TE, Mudge KM, Wood VE (2007) Functional deficit after transfer of the pronator trees for acquired radial nerve palsy. J Hand Surg Am. 32:526-530.

39. Ranter JA, Peljovich A, Kozin SH (2010) Update on tendon trans- fers for peripheral nerve injuries. J Hand Surg Am. 35:1371-1381.

40. Abrams GD, Ward SR, Friden J, Lieber RL (2005) Pronator teres is an appropriate donor muscle for restoration of wrist and thumb extension. J. Hand Surg 30A:1068-1073.

41. Mackinnon SE, Roque B, Tung TH (2007) Median to radial nerve transfer for treatment of radial nerve palsy. Case report. J Neurosurg 107(3):666-671.

42. Lowe JB 3rd, Tung TR, Mackinnon SE ( 2002) New surgical option for radial nerve paralysis. Plast Reconstr Surg 110(3):836-843.

43. Reichert P, Wnukiewicz W, Witkowski J, Bochenska A, Mizia $\mathrm{S}$, Gosk J et al (2016) Causes of secondary radial nerve plasy and results of treatment. Med Sci Monit. 22:554-562 